\title{
A Process Model of Scholarly Media Annotation
}

\author{
Liliana Melgar \\ Univ. of Amsterdam \\ Netherlands
}

\author{
Marijn Koolen \\ Huygens/ING \\ Netherlands
}

\author{
Hugo Huurdeman \\ Univ. of Oslo \\ Norway
}

\author{
Jaap Blom \\ Netherlands Inst. for Sound \\ and Vision, Netherlands
}

\begin{abstract}
Annotation has been identified as one of the "scholarly primitives", and plays a pivotal role in facilitating access to audio-visual (AV) media in a scholarly context. However, there is a lack of understanding of scholars' annotation needs and behavior. This paper is part of a group of studies aiming to understand how to improve annotation support of AV media, in order to facilitate research activities of media scholars and other scholars who make intensive use of AV media. The main findings confirm previous research discerning stages in media scholars' research processes, and indicate a great variety of research activities which occur in a non-linear order. Our studies also show that different annotation activities occur along those stages. The main contribution of this paper is a generic process model capturing AV media annotation, potentially applicable to a variety of research use cases in a scholarly context.
\end{abstract}

\section{Keywords}

time-based media; audio-visual media research; video annotation

\section{INTRODUCTION}

Scholars in the (Digital) humanities are active and motivated annotators [24]. They annotate all types of media at any level, with many layers of interpretation. Unsworth [21] identified annotating as one of the "scholarly primitives." In the case of audio-visual (AV) or time-based media, manual or semi-automatic annotation of the media content is essential, given the fact that providing fully automated access is more challenging than to textual resources [15]. Scholarly work is also often described as a process [11], where different stages occur over time (e.g., [4]). Providing support to scholarly research requires the analysis of the complex research tasks where knowledge construction is involved. These are not limited to searching and retrieving a list of results, but also other series of scholarly primitives, e.g. classifying, linking [20], comparing, sampling, illustrating, annotating [21], or writing and collaborating [18]. However, little is known about how those complex tasks are performed in the context of scholarly research where AV media is the focal point, for instance, in media and communication studies. In addition, there is a lack of system support for the different

Permission to make digital or hard copies of all or part of this work for personal or classroom use is granted without fee provided that copies are not made or distributed for profit or commercial advantage and that copies bear this notice and the full citation on the first page. Copyrights for components of this work owned by others than the author(s) must be honored. Abstracting with credit is permitted. To copy otherwise, or republish, to post on servers or to redistribute to lists, requires prior specific permission and/or a fee. Request permissions from permissions@acm.org.

CHIIR '17, March 07 - 11, 2017, Oslo, Norway

(C) 2017 Copyright held by the owner/author(s). Publication rights licensed to ACM. ISBN 978-1-4503-4677-1/17/03 . \$ \$15.00

DOI: http://dx.doi.org/10.1145/3020165.3022139 methods that this group of researchers uses in different research phases [9]. To understand how annotation should be supported along the research process of media scholars, different investigations are conducted within the CLARIAH project ${ }^{1}$, with a focus on research behavior, activities, models, and "tool" analyses. As part of these investigations, this paper presents two user studies about scholars' research processes, with a particular focus on the types of annotation-related activities. The research problem above results in the following research question: How is annotation of time-based media done in practice by media scholars, and other scholars who make intensive use of AV media, and in which stages of their research process is it used and how?

The main contributions of this paper consist of (a) extending current research on how to support different stages of the search and research process and (b) identifying the different annotation activities during the research process and the connections between them. Based on our findings and previous research, we propose a generic process model to capture annotation activities across all research stages of the analyzed use cases and highlight the main practical and conceptual implications for future system design.

\section{BACKGROUND RESEARCH}

\section{1 (Re)search as process}

Detailing the process of scholarly research is done either via "prescriptive" models used in textbooks, or discovered via empirical studies of researchers' behavior. Case [3, p. 222] lists a 'classic' view on these stages, which typically start with imagining a research question (1), followed by determining what data are needed and designing a specific study to collect it (2), choosing and implementing research methods (3), analyzing and interpreting observations (4), and considering the overall results (5). The exact nature of these stages, however, is usually not as straightforward, and may vary across disciplines. Media scholars are at the intersection of humanities and social sciences [11]. The research process of social scientists is described in 'prescriptive' models, for instance, Kendall [12], who details a series of "steps" which vary depending on the approach (inductive or deductive). Common steps in the two approaches include: problem definition, literature review, research design, data collection and analysis. In the humanities, on the other hand, the research process cannot necessarily be captured in a sequential model [11]. However, there is current empirical evidence that there are broad phases in the research process of media and communication scholars [2], and of media scholars using web archives [9]. Bron et al. [2] explored this issue by investigating a group of twenty-seven media studies researchers. The authors

${ }^{1}$ CLARIAH is project to develop a Dutch research infrastructure for Digital Humanities, see http://www.clariah.nl/ 
found common parts in the research processes, summarized in three phases with different associated activities: exploration (studying background material, developing initial research questions, initial information gathering), contextualization (revising research questions, gathering material or selecting data with a focused purpose) and presentation (organizing the data and selecting appropriate evidence to build up a case). Marsden et al. [13] briefly discuss a schematic view of the research process with AV materials in a number of humanities disciplines, which included annotation.

\subsection{Annotations and annotation behaviour}

The term "annotation" has different meanings to different communities [8]. In the literary tradition, it is used as an equivalent of "glossing.", including "marginalia," or "commonplace books" [26], often used as part of reading activities. Marshall [14] argued the need for annotation tools that support a smooth integration of annotating with reading in a digital environment. In digital humanities, annotation is regarded as one of the "scholarly primitives", which are basic activities or functions common to research across humanities disciplines, over time, and independent of theoretical orientation [21]. Palmer et al. [18] recognized notetaking as one of the cross-cutting primitives. In information processing, the term "annotation" refers to any description that is explicitly used for the purpose of future retrieval [7] (e.g. markup elements or descriptive metadata). Agosti and Ferro [1] and Haslhofer et al. [6] showed how annotations created during reading can be used as metadata. Haslhofer et al. [6], Ruvane [19] and Melgar [15] suggest a holistic view on annotation that is not limited to note-taking, but includes any form of metadata authoring (i.e., indexes, catalog records, tags, keywords, comments, notes), or even the creation of derivative documents or pieces of information derived from the initial information source. These annotations are created by any actor (expert or non-expert both in the domain and the annotation task) in the information interaction continuum, either during reading, information processing, or research. In this scope, it may be possible to study people's "information-annotation behavior" as a form of information use [15]. This broad sense of the term is conveyed in the World Wide Web Consortium (W3C) Annotation Working Group's definition of "annotation" as "any object that is associated with another object by some relationship" [23]. Current work on standards and frameworks for implementing annotation on the web include the $\mathrm{W} 3 \mathrm{C}$ candidate recommendation for the Web Annotation Data Model $^{2}$ and the "Annotating All Knowledge" coalition ${ }^{3}$. These initiatives do not necessarily focus on the needs of media scholars or annotation of $\mathrm{AV}$-media.

\subsection{Media studies research foci and use cases}

Humanities scholars are not a homogeneous group [16], and the same can be said about media scholars. Stokes (2003, as cited in [17]) indicates that media and cultural studies focus on three "strands of research": (a) industry (production and distribution of media content), (b) text (style, structure, and meaning of media forms), and, (c) audience (reception of media content). The term "use case" is frequently used in traditional system development [10] . We use the term "research use case" to refer to a type of scholarly research question and possible methods to achieve an answer to it. Research use cases can be grouped into research foci. These foci do not necessarily have epistemological implications for the domain as such, but can guide the design of better systems for supporting specific user groups. Previous work on research behavior of film and television scholars [15] identified different re-

\footnotetext{
${ }^{2}$ https://www.w3.org/TR/2016/CR-annotation-model-20161122

${ }^{3} \mathrm{https}: / /$ hypothes.is/annotating-all-knowledge
}

search foci, based on patterns in the types of research questions, data sources needed, and the analysis process. These research foci include:

1. Media aesthetics: the study of media as "texts", e.g. their genres, stylistic features, themes, or narrative structures.

2. Social history of media: the study of patterns of production, distribution, exhibition, or reception of media products (e.g. films, broadcasts, web sites) at a certain moment or place.

3. Aesthetic historiography: a combination of media aesthetics and social media history.

4. Social and cultural history: the intensive use of AV media or web data as sources of historical research.

An analysis of scholarly publications conducted in the beginning of the CLARIAH project further showed three additional foci:

5. Media representations or coverage: the study of coverage, depiction or discussion of specific events, debates, groups of people, or other realities by one specific medium.

6. Trans-media analysis: the comparison of media representations of an issue across different media.

7. Memory studies: the study of the representation of historical events with a focus on how people construct a memory of history.

\section{METHOD}

This paper is based on two user studies with media scholars in the context of the CLARIAH project. These studies looked at the scholars' research process, with a particular focus on annotation:

A full-day workshop In a hands-on setting, researchers could work with a number of digital tools for searching AV media collections which were going to be part of the infrastructure, including basic and faceted search, tools for temporal analysis, linked data exploration and viewing and annotating video interviews. Five scholars and five information specialists participated, and each media scholar teamed up with an information expert to conduct two simulated research tasks. First, a research focus was assigned to each team. The assigned foci (section 2.3) included: media aesthetics (1), aesthetic historiography (3), social and cultural history (4), media representations (5), and trans-media analysis (6).

Each researcher was familiar and had previous experience with the research focus that was assigned to her. One of the tasks consisted of conducting a research project, from beginning to end, by selecting collections and research tools, but also describing what was needed to conduct the research process at a broader level (e.g., external data, or desired functionalities of the tools). The information expert in the team had to register the steps in a template. Finally, a questionnaire about the two tasks was distributed.

Interviews At a later stage, two of the six experienced media scholars who participated in this workshop, plus a $\mathrm{PhD}$ student were interviewed about their research process, this time with a focus on their annotating and analysis activities. These interviews were one-to-one, semi-structured, and lasted between one and three hours. The interviewees' research foci included: media aesthetics (1), social and cultural history (4), and trans-media analysis (6).

The resulting data from both studies consisted of five templates (describing the research questions, "steps", datasets, needed functionalities of tools), and three interviews (audio recordings and summary transcripts). We analyzed the content in two ways: (a) clustering all research activities from both studies following their sequential order, and (b) grouping the activities identified in (a) into categories and research phases, which resulted in the final model. 


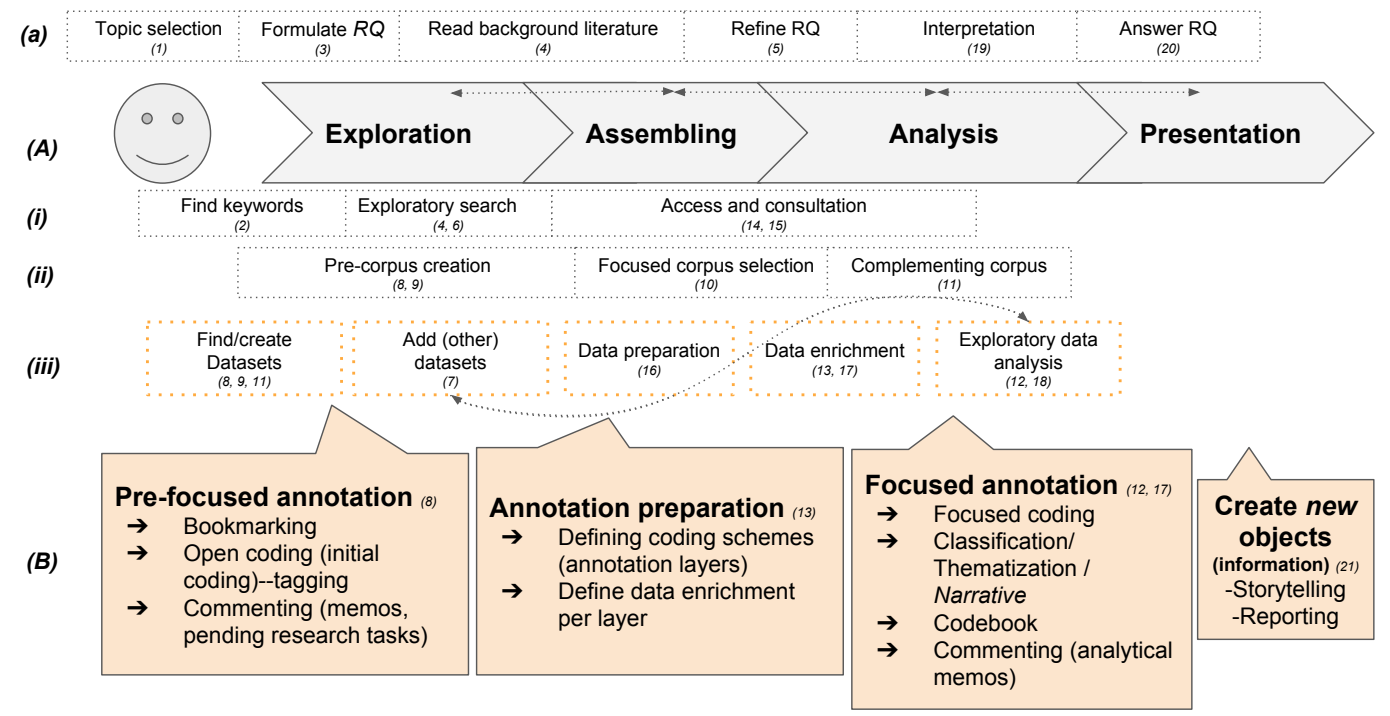

Figure 1: A process model of scholarly media annotation (numbers in parentheses correspond to Table 1)

\section{FINDINGS}

Annotation activities in media scholarly research Table 1 summarizes the 21 activities identified in our first part of the analysis. We noticed that all participants indicated a number of activities centered on the creation of a corpus and its analysis. In terms of system support, for instance, while exploring a digital collection, scholars indicated the need for support in bookmarking, selecting groups of items after using faceted search or other filtering options, memory features such as query history, selection features for AV media fragments, and the addition of manual annotations, such as comments or tags. During analytic activities, even though the participants used different terms to explain their analysis methods, they often mentioned 'coding' or 'thematization'. In this stage, they also indicated desired functionalities and/or interface features related to refined segmentation, audio transcription, automatic image/audio analysis, fragment summarization, encoding, structuring relationships between coding terms, and linking back to original sources. Some scholars described that the analysis stage included a preparatory phase in which they defined their coding schemes, and/or made their data ready for analysis (e.g, by transcribing it). Our findings also confirmed that media and AV-centered scholars do not annotate time-based media in isolation, but in relation to other media (e.g., scripts, reviews, promotional materials, etc.). We noticed similar annotation activities as those supported by specialized qualitative data analysis software (QDA), see for instance [5]).

Table 2 shows the actual order that was followed by each researcher to accomplish the research project (the numbers refer to the activities in Table 1). This confirms previous studies about the lack of a strict sequence in the research process of humanists, and media and communication scholars (similar to Ellis' study of social scientists, as cited in Case [3, p. 291]). Instead, research activities seem to be recurrent in the different research stages, but with a different degree of refinement. This is especially clear in the case of adding codes or tags to items or fragments, which is more loose in the initial exploratory stage, and more focused in the analysis stage. Likewise, some of the features above were suggested to have different purposes at different moments. For example, in initial research phases, visualization was used for exploratory data analysis. During the analysis, however, visualization was used to facilitate
Table 1: Research activities listed by participants, grouped by research stage

INITIATION/EXPLORATION

(1) Topic selection, (2) Find terms or keywords,

(3) Formulate RQ, (4) Search and read background literature,

(5) Refine RQ, (6) Explore dataset,

(7) Explore other related datasets (aka. 'contextualization')

ASSEMBLING

(8) Pre-corpus identification (identify relevant sources, define "sampling"/selection strategy),

(9) Corpus selection (or creation of datasets),

(10) Refine corpus (focused selection),

(11) Complement corpus (additional data collection, when incomplete)

ANALYSIS

(12) Pre-analysis of initial corpus,

(13) Data (corpus) preparation for analysis,

(14) 'Contextualize' corpus (find details about the items in a corpus),

(15) Access and consultation, (16) Analysis preparation,

(17) Analyze corpus,

(18) Exploratory data analysis (visualization of own corpus),

(19) Interpretation, (20) Answer RQ

\section{PRESENTATION}

(21) Present

interpretation of the patterns detected in their own corpus.

A model of the annotation process We grouped the 21 activities into categories, depicting them in a graphical conceptual representation, shown in Figure 1. The categories include: the main generic research activities in the research process (a), also represented in (A) in the form of research phases. For representing this part, we departed from the model in [2]. However, in our data we observed that a great proportion of the activities was related to data analysis. Therefore, we extended the original sequence in Bron et al. [2] by adding "analysis" as a separate research phase. We also renamed the "contextualization" phase in Bron et al. [2] to "Assembling" to be more specific. The most important part of the figure, (B), shows the annotation-related activities, which we grouped into phases, from pre-focused annotation to the creation of new information objects. We used the concepts of "pre-focused" and "fo- 
Table 2: Order of research activities listed by the participants

\begin{tabular}{l|l|l} 
Participant & Research focus & Activity order \\
\hline P1 (workshop) & Aesthetics & $1,3,2,8,14,15,17$ \\
P2 (workshop) & Media repr. & $3,8,4,9,7,10,17,18,11,18$ \\
P3 (workshop) & Trans-media & $2,3,9,13,18,16,17$ \\
P4 (workshop) & Aesthetic hist. & $3,9,4,16,17,7,11,17,18,19,21$ \\
P5 (workshop) & Cultural history & $8,7,9,12,11,15,17,19,20$ \\
P6 (interview) & Aesthetics & $3,8,4,15,14,9,10,12,13,(17,18,19)$ \\
P7 (interview) & Trans-media & $2,3,9,5,10,13,17$ \\
P8 (interview) & Memory studies & $1,3,9,11,7,12,13,17,18,19,21$
\end{tabular}

cused" from [22] to name the annotation-related stages. Indeed, previous research has found that scholars usually create their own set of semantic categories [25], which correspond to their research questions. We grouped the other research activities in four layers: (i) connected to information seeking and searching (not necessarily system-mediated), (ii) broader stages related to the corpus, and (iii) more specific data processing related activities around the creation and annotation of those corpora.

\section{DISCUSSION AND CONCLUSION}

The user studies described in this paper confirm that there are discernible, though not strictly sequential, research activities in the research process of media scholars and other AV-centered scholars. In addition, annotation activities seem to have a pivotal supporting function, varying across research stages. Although still in a preliminary stage, the annotation model introduced in this paper contributes to an improved conceptual understanding of information annotating behavior in media research as a process.

These findings have both practical and theoretical implications for future system design applicable to scholarly research. At a practical level, future search systems should offer annotation support. This support can range from basic (e.g. facilitating corpus building), to more complex (e.g. for dynamic analytical activities), or even for the entire research process from corpus creation to data analysis and writing. Most of these tasks are currently supported by separate specialised software, (e.g, QDA packages, or authoring platforms), but the annotations they produce are hardly interoperable. At a theoretical level, the conceptual model presented here has implications for the design of annotation standards and frameworks. For instance, the W3C's Web Annotation Data model, at this point, proposes the concept motivation as a way to represent the intent behind the creation of an annotation. However, the terms seem to indicate activities, or tasks (i.e. sequences of activities) rather than the actual purpose (e.g., analysis). Besides, it does not seem to take into account other ontologies related to research activities (e.g., TaDiRAH) ${ }^{4}$. More research needs to be done to determine whether a phase of the research process should be considered part of the model, or how to handle refinements of annotations for conveying stage-related information. Future work should also provide more details about the requirements for the annotated objects (targets) when there is a need to take into account their structural composition. Finally, because annotations are created by agents with different expertise levels, the Web Annotation Data Model should specify both domain and annotation expertise of the agents involved. This information is essential for supporting collaborative and more varied ways of annotation.

\footnotetext{
${ }^{4}$ http://tadirah.dariah.eu/vocab/index.php
}

\section{References}

[1] M. Agosti and N. Ferro. Annotations as Context for Searching Documents, pages 155-170. Springer Berlin Heidelberg, 2005.

[2] M. Bron, J. Van Gorp, and M. Rijke. Media studies research in the data-driven age: How research questions evolve. J. Am. Soc. Inf. Sci. Tec., 2015.

[3] D. O. Case. Looking for information: A survey of research on information seeking, needs and behavior. Emerald Group Publishing, 2016.

[4] C. M. Chu. Literary critics at work and their information needs: A research-phases model. Libr. Inform. Sci. Res., 21(2):247-273, 1999.

[5] G. R. Gibbs. Using Software in Qualitative Analysis. In The SAGE Handbook of Qualitative Data Analysis, pages 277-294. SAGE Publications Ltd, 2014.

[6] B. Haslhofer, W. Jochum, R. King, C. Sadilek, and K. Schellner. The LEMO annotation framework: weaving multimedia annotations with the web. Int. J. on Digital Libraries, 10(1):15-32, 2009.

[7] L. Hollink. Semantic annotation for retrieval of visual resources. Doctoral Dissertation, Vrije Universiteit, 2006.

[8] J. Hunter. Collaborative semantic tagging and annotation systems. Annu. Rev. Inform. Sci., 43(1):1-84, 2009.

[9] H. C. Huurdeman. Supporting the Complex Dynamics of the Information Seeking Process. Doctoral thesis, University of Amsterdam, 2017, forthcoming.

[10] I. Jacobson, I. Spence, and B. Kerr. Use-case 2.0. Commun. ACM, 59 (5):61-69, 2016

[11] K. B. Jensen. The complementarity of qualitative and quantitative methodologies in media and communication research. In A handbook of media and communication research, pages 283-301. Routledge, London [etc.], 2nd edition, 2012.

[12] D. E. Kendall. Sociology in our times: the essentials. Thomson/Wadsworth, Belmont, CA, 2006.

[13] A. Marsden, A. Mackenzie, A. Lindsay, H. Nock, J. Coleman, and G. Kochanski. Tools for searching, annotation and analysis of speech, music, film and video - A survey. Literary and Linguistic Computing, 22(4):469-488, 2007.

[14] C. C. Marshall. Annotation: from paper books to the digital library. In Proceedings of the Second ACM International Conference on Digital Libraries, pages 131-140. New York: ACM, 1997.

[15] L. Melgar. From social tagging to polyrepresentation (doctoral thesis). A study of expert annotating behavior of moving images. University Carlos III of Madrid, 2016.

[16] U. Must. Alone or together: examples from history research. Scientometrics, 91(2):527-537, 2012

[17] J. Noordegraaf. Computational research in media studies: methodological implications. Kwalon, 21(1):52-59, 2016.

[18] C. L. Palmer, L. C. Teffeau, and C. M. Pirmann. Scholarly information practices in the online environment: themes from the literature and implications for library service development. Technical report, OCLC Research, 2009.

[19] M. B. Ruvane. Annotation as process: A vital information seeking activity in historical geographic research. In Proceedings ASIST, volume 42, 2005.

[20] R. Sanderson, P. Ciccarese, and H. Van de Sompel. Designing the W3C open annotation data model. In Web Science Proceedings, pages $366-375,2013$.

[21] J. Unsworth. Scholarly Primitives: what methods do humanities researchers have in common, and how might our tools reflect this? Humanities Computing: formal methods, experimental practice, May 13, 2000, London, UK.

[22] P. Vakkari. A theory of the task-based information retrieval process: a summary and generalisation of a longitudinal study. J. Doc., 57(1): 44-60, 2001

[23] W3C. Collaboration, knowledge representation and automatability, 1995.

[24] N.-O. Walkowski and E. T. E. Barker. Digital humanists are motivated annotators. In Digital Humanities 2014, Laussane, Switzerland, 2014.

[25] S. Westman. Image users' needs and searching behaviour. In Information Retrieval: Searching in the 21st Century; Human Information Retrieval, chapter 4, pages 63-83. 2009.

[26] M. Winget. A meditation on social reading and its implications for preservation. Preservation, Digital Technology \& Culture, 42(1):39_ 52, 2013. 